Toimela, T. Effective Interaction Potential in the Deuterium Plasma and Multiple Resonance Scattering. in Eleventh International Conference on Condensed Matter Nuclear Science. 2004. Marseille, France.

\title{
Effective Interaction Potential in the Deuterium Plasma and Multiple Resonance Scattering
}

\author{
T. Toimela \\ Vaasa Polytechnic, Wolffintie 30, 65200 Vaasa, Finland \\ www.puv.fi; E-mail: tuomo.toimela@puv.fi
}

\begin{abstract}
The effective interaction potential for charged particles is calculated in the deuterium plasma formed in the surface region of the palladium cathode in the electrolysis of heavy water. It is shown that the Coulomb potential is overscreened producing, at certain distances, an attractive potential between deuterium nuclei pairs and also between the deuterium nuclei and the surface atoms. This behavior of the effective potential in the deuterium plasma can be regarded as a counterpart of the Friedel oscillations of the electron gas at zero temperature. Because of this attractive potential, there are bound states for the deuteron pairs as well as for the surface atoms and deuterons. In these bound states the equilibrium distance between the nuclei is of the order $0.15-0.2 \AA$ depending on the number density of deuterons. The fusion rate is calculated for the bounded deuteron pairs and it is found to be of the order $\lambda \approx 10^{-22} 1 / \mathrm{s}$ per deuteron pair for the highest deuteron densities. Furthermore, it is shown that larger fusion rates are possible for the deuterons bound to the surface atoms. This may arise by a process, where the trapped deuterons share the released energy collectively via a proposed Multiple Resonance Scattering (MRS) mechanism. It is then found that the ${ }^{4} \mathrm{He}$-channel is the overwhelmingly dominant fusion channel. Moreover, the appearance of the transmutation processes in this MRS procedure is briefly discussed.
\end{abstract}




\section{Introduction}

The experimental results concerning condensed matter nuclear science, starting from the first announcement of cold fusion [1], are very extraordinary. The idea that there could be nuclear processes caused by the condensed matter environment is astonishing. Furthermore, the experimental results showing that practically no radiation is obtained and that the branching ratios are not the usual ones obtained in the collision experiments of deuterons are even more astonishing features. Including the claims that there are also occurring transmutation processes of heavier nuclei, which should be even more improbable reactions, it is not a surprise that this area of research has been mostly ignored by the main physics community.

However, the aim of this article is to show that those extraordinary features may be explained without any new unknown physical theories, just with reasonable assumptions and correct treatment of the well-established quantum many-body theory. We assume that the deuterium ions reaching the cathode will form (together with the electrons around) the deuterium plasma in the surface region of palladium (or other metal used as the cathode). The effective interaction in this plasma and its consequences will be examined in this work.

This article is organized as follows: In the next chapter the effective potential in the deuterium plasma is calculated. It is shown that the deuteron pairs will acquire an attractive potential, which sustain bound states. The fusion rate in these pairs will be calculated and a low-level neutron emission is shown to arise. The details of the calculations of that chapter will be published elsewhere [2]. In Section 3, the deuterons trapped by the surface atoms are considered. A multiple resonance scattering mechanism is proposed, which is shown to lead to predictions that are consistent with the main experimental results in the condensed matter nuclear science. In the last section an outlook for further studies will be given.

\section{Effective potential}

Following the standard electron gas calculations in solids [3], the Hamiltonian in the plasma composed of deuterons and electrons can be written as the sum of six terms:

$$
\mathrm{H}=\mathrm{H}_{\mathrm{el}}+\mathrm{H}_{\mathrm{d}}+\mathrm{H}_{\mathrm{el}-\mathrm{d}}+\mathrm{H}_{\mathrm{b}}+\mathrm{H}_{\mathrm{el-b}}+\mathrm{H}_{\mathrm{d}-\mathrm{b}},
$$

where $\mathrm{H}_{\mathrm{el}}\left(\mathrm{H}_{\mathrm{d}}\right)$ is the Hamiltonian for the electrons (deuterons) respectively (containing the kinetic energy term plus the Coulomb interaction). $\mathrm{H}_{\mathrm{el}-\mathrm{d}}$ contains the Coulomb interaction between deuterons and electrons. $\mathrm{H}_{\mathrm{b}}$ is the interaction energy between the background surface atoms. $\mathrm{H}_{\mathrm{el}-\mathrm{b}}$ and $\mathrm{H}_{\mathrm{d}-\mathrm{b}}$ are the interaction energies between the surface atoms and electrons and deuterons, respectively. Assuming uniform distribution 
for the surface atoms, the three last energies are just pure numbers and can be ignored here. Beyond these trivial contributions the quantity containing the physically interesting interaction, is given by the effective interaction, obtained in the ring-diagram approximation (random phase approximation).

$$
\mathrm{U}_{\mathrm{eff}}=\frac{\mathrm{U}_{0}(\omega, \mathbf{q})}{1-\Pi_{0}(\omega, \mathbf{q}) \mathrm{U}_{0}(\omega, \mathbf{q})}
$$

where $\omega$ and $\mathbf{q}$ are the angular frequency and the wave vector, respectively. The Coulomb interaction in the momentum space, $\mathrm{U}_{0}(\omega, \mathbf{q})$ is given by

$$
\mathrm{U}_{0}(\omega, \mathbf{q})=\frac{e^{2}}{\varepsilon_{0} \mathbf{q}^{2}}
$$

corresponding to the usual Coulomb potential in coordinate space:

$$
\mathrm{U}(\mathrm{r})=\frac{e^{2}}{4 \pi \varepsilon_{\mathrm{o}} \mathrm{r}}
$$

(The standard SI-units are used here.) In Eq. (2) $\Pi_{0}(\omega, \mathbf{q})$ is the polarization insertion calculated at the first loop level. In the following we shall consider only the static limit of the interaction, $(\omega \rightarrow 0)$. In the deuterium plasma both the deuteron and the electron fields have to be regarded dynamical variables (unlike the lattice atoms in the electron gas calculations of metal). Hence, the polarization insertion has two terms, corresponding to both the electron and the deuteron loops. These can be written as [3]

$$
\begin{aligned}
\Pi_{0}(0, q) & =\Pi_{0}{ }^{(e)}(0, q)+\Pi_{0}{ }^{(d)}(0, q) \\
& =2 \int \frac{d^{3} p}{(2 \pi)^{3}} \frac{n^{F_{p+q}}-n^{F}}{E_{\mathbf{p}+\mathbf{q}}-E_{\mathbf{p}}}+3 \int \frac{d^{3} p}{(2 \pi)^{3}} \frac{n^{B}{ }_{\mathbf{p}+\mathbf{q}}-n^{B} \mathbf{p}}{E_{\mathbf{p}+\mathbf{q}}-E_{\mathbf{p}}},
\end{aligned}
$$

where $\mathrm{n}_{\mathbf{p}}^{\mathrm{F}} \quad\left(\mathrm{n}_{\mathrm{p}}^{\mathrm{B}}\right)$ is the Fermi (Bose) distribution for electron (deuteron) loop, respectively. $E_{p}$ is the energy corresponding to the momentum $\hbar p$. It can be approximated to be the kinetic energy of a free particle. Note that the RPA approximation with the free particle energy spectrum used here is the same that has been extensively used in the standard electron gas calculations [3] producing (at least) a qualitative agreement with experiments.

Consider the first term (the electron loop) in Eq. (5). The electron gas in metal is highly degenerate at the room temperature and consequently a reasonable approximation is to assume the electron states to be filled up to the Fermi energy and to be empty above it. The outcome is then the well-known Thomas-Fermi screening, which, however, has too long screening length to play any role in the fusion processes [4]. Hence, we concentrate 
on the deuteron loop, the second term in Eq. (5). (We can assume alternatively that the electrons form a uniform average background.)

If the temperature is high enough $(\approx 300 \mathrm{~K})$ and the deuterium plasma is not too dense, the Bose distribution can be replaced by the classical Maxwell distribution. The polarization insertion (corresponding to the deuteron loop) can then be written as [3]

$$
\Pi_{0}^{(d)}(0, q)=-3 e^{\mu / k T} \frac{m^{2} k T}{\pi^{2} q \hbar^{4}} \int_{0}^{\infty} d x e^{-x^{2}} \ln \left|\frac{x+b}{x-b}\right|,
$$

where $\mathrm{b}$ is given by $\mathrm{b}(\mathrm{q})=\hbar \mathrm{q} / \sqrt{8 \mathrm{mkT}}$.

The chemical potential $\mu$ of the deuterons can be related to their number density, $\mathrm{n}$. If we assume [consistently with the energy approximation used in Eq. (5)] that the deuterons form an ideal classical gas, the number density of deuterons is

$$
\mathrm{n}=3 \mathrm{e}^{\mu / \mathrm{kT}}\left(\frac{\mathrm{mkT}}{2 \pi \hbar^{2}}\right)^{3 / 2}
$$

Using this result we can rewrite Eq. (6) as

$$
\begin{aligned}
\frac{e^{2}}{\varepsilon_{\mathrm{o}}} \Pi_{0}^{(\mathrm{d})}(0, \mathrm{q}) & =-\frac{4 \pi \alpha \hbar \mathrm{cn}}{\mathrm{kT}} \mathrm{I}(\mathrm{b}) \\
& =-\frac{4 \pi \alpha \hbar \mathrm{cn}}{\mathrm{kT}} \frac{1}{\sqrt{\pi} \mathrm{b}} \int_{0}^{\infty} \mathrm{dxx} \mathrm{x}^{-\mathrm{x}^{2}} \ln \left|\frac{\mathrm{x}+\mathrm{b}}{\mathrm{x}-\mathrm{b}}\right|,
\end{aligned}
$$

where $\alpha$ is the fine structure constant. The limits of the integral I(b) can be easily found [3]:

$$
\mathrm{I}(0)=1 \quad \text { and } \quad \mathrm{I}(\mathrm{b}) \approx \frac{1}{2 \mathrm{~b}^{2}}=\frac{4 \mathrm{mkT}}{\hbar^{2} \mathrm{q}^{2}} \quad(\mathrm{q} \rightarrow \infty)
$$

The function (8) should now be inserted into the effective interaction of Eq. (2), which in the coordinate space is

$$
\mathrm{U}_{\text {eff }}(\mathrm{r})=\frac{e^{2}}{\varepsilon_{\mathrm{o}}} \int \frac{\mathrm{d}^{3} \mathrm{q}}{(2 \pi)^{3}} \frac{\mathrm{e}^{\mathrm{i} \mathbf{q} \cdot \mathrm{r}}}{\mathrm{q}^{2}-\frac{\mathrm{e}^{2}}{\varepsilon_{\mathrm{o}}} \Pi_{0}(0, \mathrm{q})}
$$

This effective potential represents the modification of the Coulomb's law by the medium composed of the electrons, the deuterons and the background atoms in the surface region. 
If the polarization insertion in Eq. (10) is replaced by its low momentum limit, the wellknown Debye screening is obtained. The inverse of the screening length squared will be then

$$
\mathrm{q}_{\mathrm{D}}^{2}=-\frac{e^{2}}{\varepsilon_{\mathrm{o}}} \Pi_{0}^{(\mathrm{d})}(0,0)=\frac{4 \pi \alpha \hbar \mathrm{c} \mathrm{n}}{\mathrm{kT}}
$$

This gives the usual Yukawa-type screening of the Coulomb potential

$$
\mathrm{U}_{\text {eff }}^{(0)}(\mathrm{r})=\alpha \hbar \mathrm{c} \frac{\mathrm{e}^{-\mathrm{q}_{\mathrm{D}} \mathrm{r}}}{\mathrm{r}}
$$

The inverse of the screening length $\mathrm{q}_{\mathrm{D}}$ can be calculated provided that the number density of the deuterons is given. The density of the deuterons in the surface region of the palladium must exceed the corresponding density inside the metal (for the deuterons to be able to diffuse into the lattice structure of the metal). How much larger it will be is not known. It has been proposed that in the surface region the deuterium to palladium ratio, $\mathrm{D} / \mathrm{Pd} \geq 2.0$ [5]. If we take the modest estimate assuming the number density of the deuterons to be just twice the normal number density of bulk palladium $\left(7.0 \times 10^{28} 1 / \mathrm{m}^{3}\right)$, the screening length then becomes $1 / \mathrm{q}_{\mathrm{D}}=3.2 \times 10^{-12} \mathrm{~m}$. Although this length is much smaller than the screening length obtained from the electron loop calculation [4], it would still be too large to allow any fusion process to occur, if Eq. (12) were a sufficient approximation to the effective potential given by Eq. (10). Classically, the minimum distance of deuterons scattered by each other with thermal energies corresponding to the room temperature $(300 \mathrm{~K})$ is about $0.2 \AA$ if the Yukawa potential of Eq. (12) is used. The amount of time spent by the deuterons in such distances, however, would be far too short to produce detectable tunneling rates.

However, it is evident that the usual approximation, in which the polarization insertion is replaced by its low momentum limit, is not sufficient here. This can be seen from the fact that the expansion parameter $b=b(q)$ in Eq. (8) is of order 3 when $q \approx q_{D}$. Hence Eq. (10) has to be evaluated with greater accuracy by inserting the whole polarization function given by Eq. (8). To do this, numerical calculations have to be performed. The results (obtained by using MathCAD) are shown in Fig. 1, the deuteron density being $(1.4-2.8) \times 10^{29} 1 / \mathrm{m}^{3}$ (the deuterium to palladium ratio ranging from 2.0 to 4.0 ).

The momentum dependence of the polarization insertion changes the Yukawa-type screening potential to one having a minimum. This potential gives an attraction between the positively charged deuterons. The classical equilibrium distance of the deuterons given by this potential is $0.16-0.20 \AA$ for the deuteron densities mentioned above. On the other hand the (classical) minimum distance of the deuterons when they are not in a bound state but are scattered by each other with thermal energies varies in this density range from $0.12 \AA$ to $0.15 \AA$. 
The potential dip of this effective potential (arising from the influence of the medium to the Coulomb law) is too small to be able to bind electrons. However, by solving (numerically) the Schrödinger equation for more massive deuterons, one finds that these potential dips sustain two $(\ell=0)$ bound states. The binding energy for the ground state varies from $2.5 \mathrm{eV}$ to $3.7 \mathrm{eV}$, while for the excited state the binding energy varies from $0.6 \mathrm{eV}$ to $1.0 \mathrm{eV}$, at the deuteron density interval quoted above. These energy states may provide a possibility to test experimentally the validity of the idea proposed here by studying the absorption of laser light the wavelength of which corresponds to the difference between the ground and the excited states.

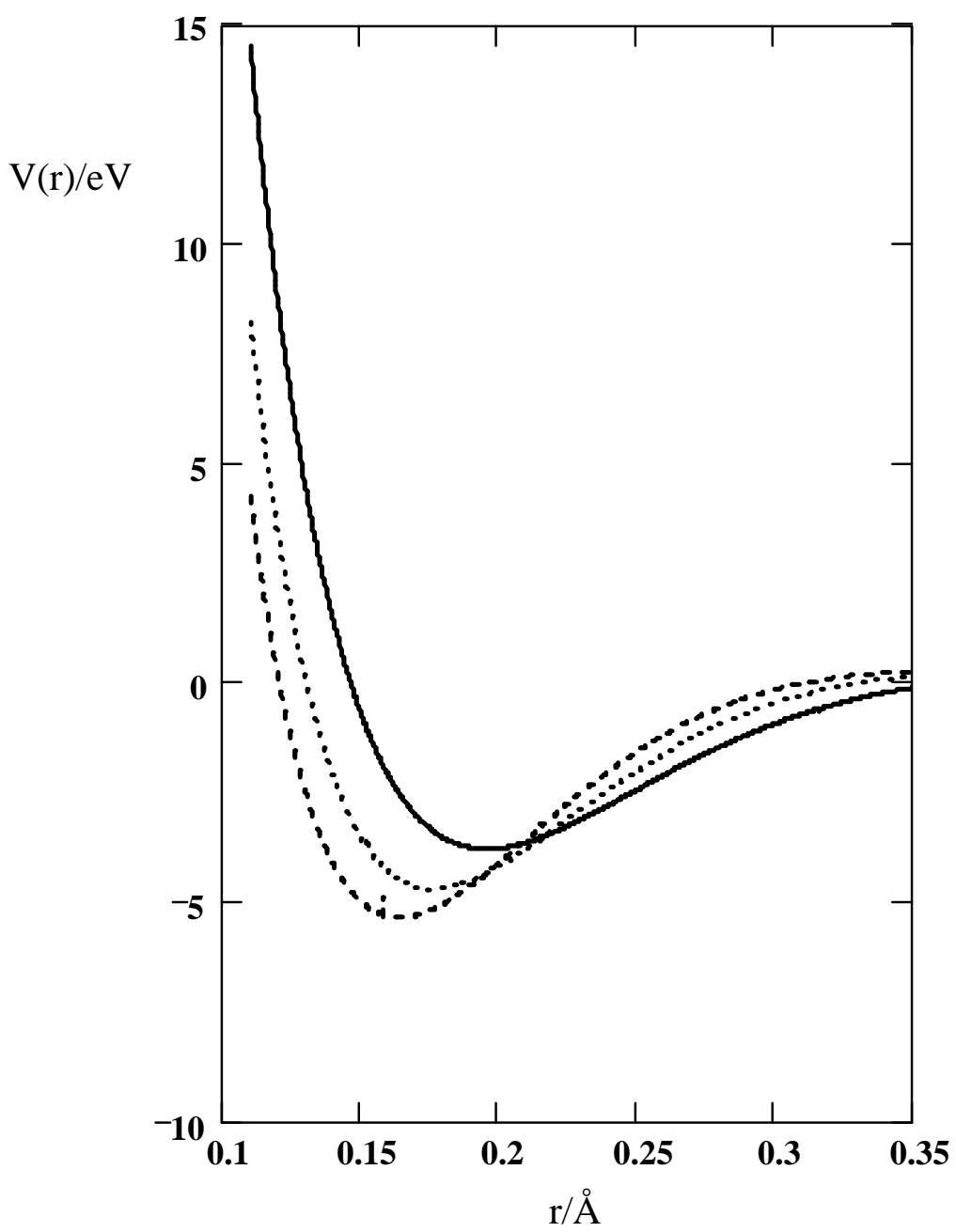

Figure 1. The effective interaction potential between deuterons as a function of their mutual distance. The solid, dotted and dashed lines correspond to the deuterium to palladium ratio $\mathrm{D} / \mathrm{Pd}=2.0,3.0$ and 4.0 respectively. 
The screening potential found here can be considered as a counterpart of the Friedel oscillations in the zero-temperature electron gas. The crucial difference is that the Friedel oscillations do not provide bound states for the electrons, but the effective potential in this deuterium plasma bind the massive deuterons. Similar behavior for the effective interaction has been obtained also by other studies [6] although leading to somewhat larger equilibrium distances.

The fusion rate for deuteron pairs in a bound state can be calculated from the value of the relative wave function $\psi_{\text {rel }}\left(\mathbf{r}_{1}-\mathbf{r}_{2}\right)$ of the two deuterons at zero distance (or strictly speaking within the nuclear interaction range, which we will use $3 \mathrm{fm}$ ). The rate is then given by

$$
\lambda=\mathrm{K}\left|\psi_{\text {rel }}(0)\right|^{2} \quad .
$$

The fusion constant $\mathrm{K}$ for the ${ }^{3} \mathrm{He}$ process, $\mathrm{D}(\mathrm{d}, \mathrm{n})^{3} \mathrm{He}$ is known to be $1.48 \times 10^{-22} \mathrm{~m}^{3} / \mathrm{s}$ being equal to the fusion constant for the tritium process $\mathrm{D}(\mathrm{d}, \mathrm{p}) \mathrm{T}$.

In the numerical calculation the subroutine "Odesolve" in MathCAD is used to solve the Schrödinger equation to obtain the relative wave function. The fusion rate is then obtained from Eq. (13). Due to the stiffness of the differential equation quite many intermediate steps (of order 10000) are required for accurate results for the wave function. The calculation reveals that when the deuteron pair is in the ground state the fusion rate for $\mathrm{D} / \mathrm{Pd}=2,3$ and 4 are $\lambda \approx 10^{-26}, 10^{-24}$ and $10^{-22} 1 / \mathrm{s}$, respectively.

If light water is used instead of heavy water, similar behavior for the effective potential takes place. (Note that the Maxwell-Boltzmann distribution is also applicable for the protons. Although the electrons are degenerate, the more massive protons are not, at the room temperature and at the densities quoted above.) However, because the low energy limit of the S-function for the reaction $\mathrm{P}(\mathrm{p}, \gamma) \mathrm{D}$ is 23 order of magnitude smaller [7] than that of the deuteron reactions $\mathrm{D}(\mathrm{d}, \mathrm{n})^{3} \mathrm{He}$ and $\mathrm{D}(\mathrm{d}, \mathrm{p})^{3} \mathrm{~T}$, the rate for the proton-proton reaction is far too low to be detectable.

Returning to the deuteron fusion, to find the fusion rate per second and per unit surface area, the thickness of the active deuterium plasma in the surface region and also the fraction of the deuterons being in the bound state must be known. If the thickness of the active surface region is about $1000 \mathrm{~nm}$ and if one tenth of the deuterons are bound [2], the fusion rates for $\mathrm{D} / \mathrm{Pd}=2,3$ and 4 are, respectively, about $10^{-7}, 10^{-5}$ and $10^{-4}$ reactions per second and per square centimeter. The highest value of these fusion rates arising from the deuteron pairs is in agreement with the low-level neutron emission results reported by S. E. Jones et al. [8], but cannot explain the excess heat and excess ${ }^{4} \mathrm{He}$ claimed in other experiments.

The order of magnitude character of our one-loop calculation for the fusion rate should be emphasized. Although the deuteron densities and the thickness of the surface plasma 
quoted above can be criticized, the sensitivity of the nuclear overlapping and consequently the fusion rate on the equilibrium distance implies that the claimed level of the neutron emission may be easily explained by the exact effective potential.

\section{Nuclear reactions in the deuteron trap}

In the previous chapter we note that the order of magnitude of the fusion rate in the bound deuteron pairs is consistent with the low-level neutron emission obtained experimentally. However, there is a possibility how this effective attraction can cause considerable larger fusion rates. Note that the effective interaction discussed in Section 2 is also valid between the surface atoms and the deuterons. Compared to the case of the deuteron pairs, the polarization insertion is unchanged, while the effective interaction as a whole has to be multiplied by the atom charge number Z. Consequently, the classical equilibrium distance between the surface atom and the deuteron will be the same as for the deuteron pairs. However, the strength of the potential will be $\mathrm{Z}$ times the strength of the potential between the deuterons. (If the shielding of the core electrons are taken into account, the nuclear charge $\mathrm{Ze}$ should be replaced by an effective charge $\mathrm{Z}_{\text {eff }}(\mathrm{r}) \mathrm{e}$, which will reduce also the strength of the attraction potential. This does not, however, change the picture qualitatively.) The binding energies of the deuterons bound to the surface atoms by this effective interaction are anyway large enough that the bound deuterons are stable against neutralization. (The neutralization can take place by multiparticle interaction. However, the rate for such neutralization processes will be small.)

The deuterons bound to the surface atoms of the cathode metal are not necessarily bound to a single surface atom but their wave function may be distributed to an extended potential of several surface atoms. In this way a group of the surface atoms can form a deuteron trap. As the cathode metal is loaded by deuterium, the number of the deuterons bound by these surface traps will increase. The extension of these surface traps can depend sensitively and in an unknown manner on several factors, like the smoothness of the surface, lattice impurities and defections and even on the chemical composition of the electrolyte. It is possible that, when the deuterium loading (in the surface region) is very low, the traps are formed by only one single surface atom, but when the deuterium loading increases, the embedded deuterons can provide "bridges" joining the attractive potentials of two adjacent surface atoms, leading to more extended traps.

As we know neither the dimensions of the traps nor the wave function of the ground state of the deuterons in these traps, we have to proceed at a very general level, in order to extract the main physical consequences of these deuteron traps.

Consider the transition from $\mathrm{N}+1$ trapped deuterons to the final state consisting free deuterons and the fusion product(s). There will be $\mathrm{N}$ particles in the final state, if we

consider ${ }^{4} \mathrm{He}$-channel, and $\mathrm{N}+1$ particles for tritium and ${ }^{3} \mathrm{He}$-channels. The transition rate is given by the golden rule 


$$
\lambda=\frac{2 \pi}{\hbar}\left|\left\langle\Psi_{\text {free }}|\hat{\mathrm{H}}| \Psi_{\text {trap }}\right\rangle\right|^{2} \rho
$$

where $\rho$ is the density of the states given by

$$
\rho=\delta\left(\mathrm{E}_{\mathrm{f}}-\mathrm{E}_{\mathrm{i}}\right)\left(\frac{\mathrm{V}}{(2 \pi)^{3}}\right)^{\mathrm{N}} \prod_{\mathrm{j}=1}^{\mathrm{N}} \mathrm{d}^{3} \mathrm{p}_{\mathrm{j}}
$$

Note that the conservation of momentum does not require here the existence of a photon in the final state in the ${ }^{4} \mathrm{He}$-channel, because the momentum is transferred by the other deuterons in the final state. We regard here the initial deuterons as free particles confined in the trap volume $\mathrm{V}_{0}$. Their momenta are negligible when compared to the momenta of the final state particles. As the Hamiltonian that is responsible for the nuclear reaction, we use the Fermi pseudopotential

$$
\hat{\mathrm{H}}=-\frac{4 \pi \hbar^{2}}{\mathrm{~m}} \mathrm{a}_{\text {nucl }} \delta(\mathbf{r})
$$

where $\mathrm{a}_{\text {nucl }}$ is the nuclear scattering length. We can assume that the nuclear scattering length for the ${ }^{4} \mathrm{He}$-channel and for the tritium and ${ }^{3} \mathrm{He}$-channels are of the same order due to the absence of the extra electric coupling to the photon field in the ${ }^{4} \mathrm{He}$-channel here. For the elastic interaction between deuterons sharing the released energy we use similar pseudopotential as Eq. (16) the scattering length for those interactions being $\mathrm{a}_{\mathrm{el}}$. The process, where the energy is collectively distributed among all the deuterons in the trap, contains N-1 elastic interaction between the deuterons (in the tree-diagram level). Hence, the rate will be of form

$$
\begin{aligned}
\lambda=\left(\frac{4 \pi \hbar \mathrm{a}_{\text {nucl }}}{\mathrm{m}}\right)^{2}\left|\psi_{\text {rel }}(0)\right|^{2}\left(\frac{4 \pi \hbar \mathrm{a}_{\mathrm{el}}}{\mathrm{m} \sqrt{\mathrm{V}_{0}}}\right)^{2(\mathrm{~N}-1)} \\
\quad \times \int \frac{\mathrm{d}^{3 \mathrm{~N}} \mathrm{p}}{(2 \pi)^{3 \mathrm{~N}}}\left(\prod_{\mathrm{i}=1}^{\mathrm{N}-1} \mathrm{G}\left(\omega_{i}, \mathrm{q}_{\mathrm{i}}\right)\right)^{2} \frac{(2 \pi)^{4}}{\mathrm{~V}_{0}} \hbar \delta\left(\mathrm{E}_{\mathrm{i}}-\mathrm{E}_{\mathrm{f}}\right) \delta\left(\mathbf{p}_{\mathrm{f}}\right)
\end{aligned}
$$

In Eq. (17) the functions $\mathrm{G}\left(\omega_{\mathrm{i}}, \mathrm{q}_{\mathrm{i}}\right)$ are the propagators corresponding to the virtual lines (the spin indices have been deleted for simplicity).

$$
\mathrm{G}\left(\omega_{\mathrm{i}}, \mathrm{q}_{\mathrm{i}}\right)=\frac{1}{\omega_{\mathrm{i}}-\frac{\hbar \mathrm{q}_{\mathrm{i}}^{2}}{2 \mathrm{~m}}-\frac{\mathrm{i}}{2} \Gamma / \hbar},
$$

where $\Gamma$ is the line width corresponding the decay of the virtual deuteron to the lower energy states. $\omega_{\mathrm{i}}$ and $\mathrm{q}_{\mathrm{i}}$ are the frequency and the wave vector of the virtual lines 
(depending on the final state wave vectors $\mathbf{p}$ in such a way that the frequency and the momentum are conserved at all the verteces in the Feynman diagram).

As we shall see below, the dominant contribution to the transition matrix arises from the diagrams of the type, where the energy released is distributed most effectively (so that the energies of the virtual particles split consecutively by interactions with the internal state deuterons). That kind of a diagram is shown in Fig. 2.

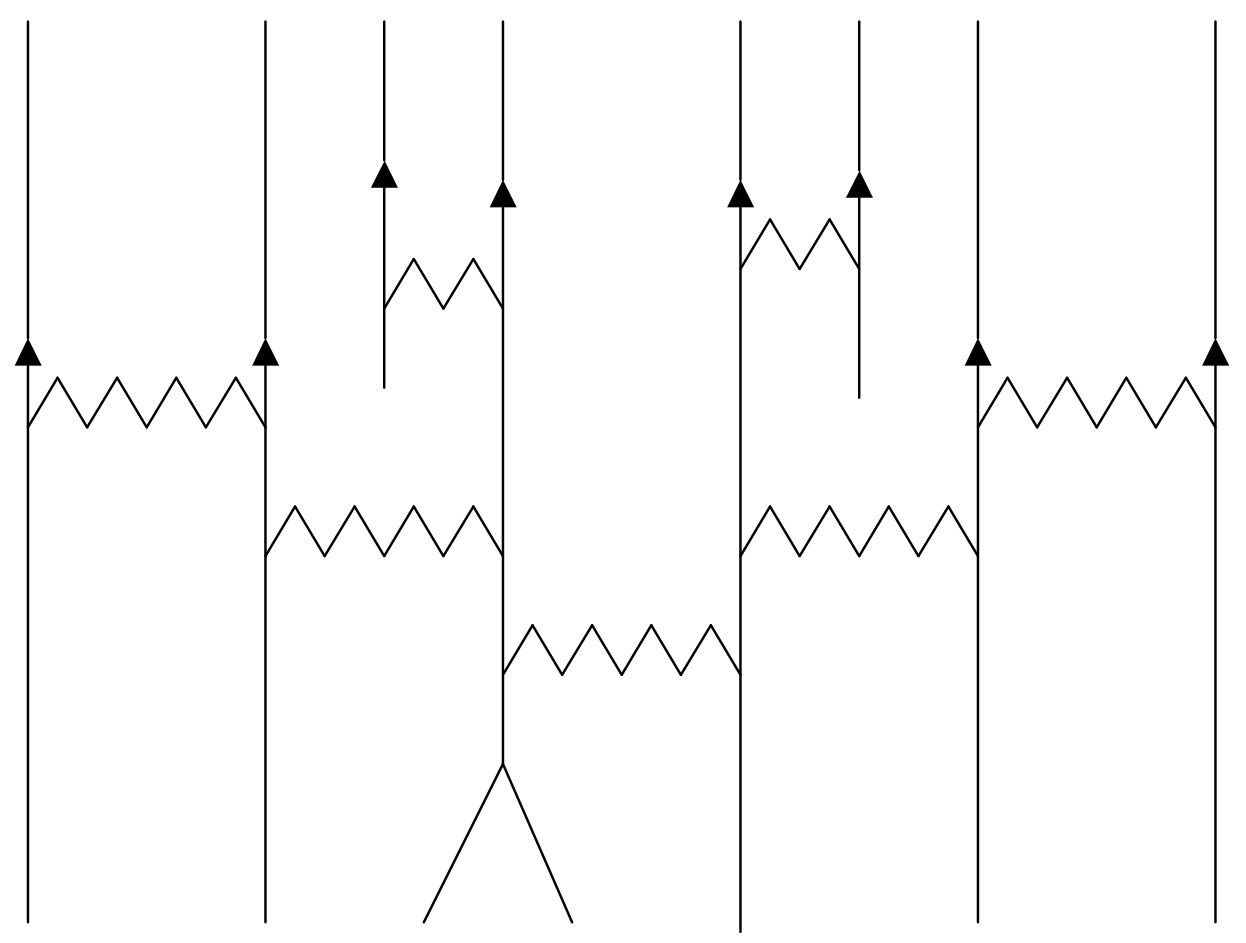

Figure 2. The type of the diagrams having largest contribution to the fusion rate.

Now, consider a virtual deuteron that interacts with an initial state deuteron so that both of them become free particles at the final state. The conservation of the energy and the momentum determine that the propagator of the virtual particle, Eq. (18), has to be

$$
\mathrm{G}\left(\omega_{\mathrm{i}}, \mathrm{q}_{\mathrm{i}}\right)=\frac{1}{\frac{\hbar \mathrm{p}^{2}}{2 \mathrm{~m}}+\frac{\hbar \mathrm{q}^{2}}{2 \mathrm{~m}}-\frac{\hbar(\mathbf{p}+\mathbf{q})^{2}}{2 \mathrm{~m}}-\frac{\mathrm{i}}{2} \Gamma / \hbar}
$$

where $\mathbf{p}$ and $\mathbf{q}$ are the wave vectors of the two final state deuterons. From Eq. (19) we note that there will be a resonance, if the final state momenta are perpendicular to each other. Furthermore, there will be multiple resonances, if the final state momenta are such 
that after every vertex the momenta of the two (virtual or real) particles are perpendicular to each other. For this multiple resonance scattering to occur, N-3 components of the final state wave vectors are restricted to be small. Moreover, the conservation of momentum gives three extra constraints. For the diagram shown in Fig. 2, the momenta of the internal and final state particles are shown in Fig. 3 for this multiple resonance situation. Note that in Fig. 3 the two first momenta have to be opposite to each other due to the conservation of momentum.

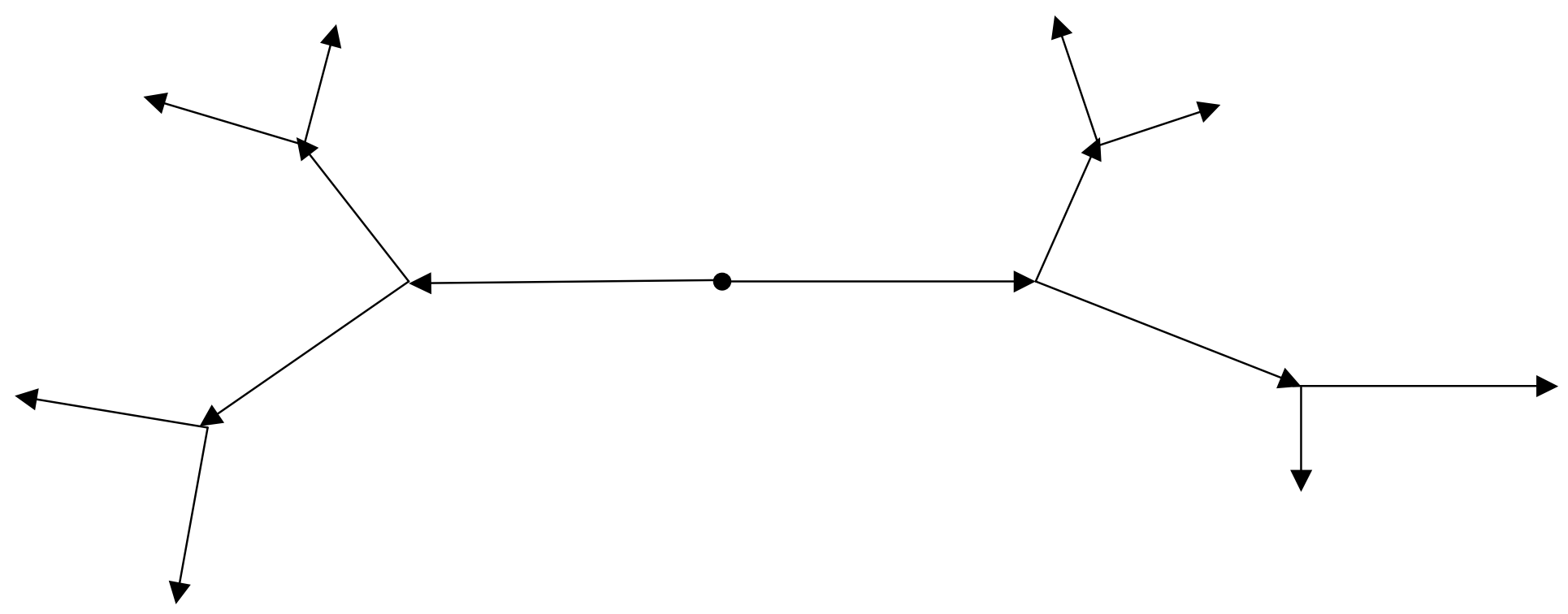

Figure 3. The momenta of the virtual and final state particles in the multiple resonance scattering corresponding to the diagram in Fig.2.

Assume that we integrate over the N-3 small components of the wave vectors that are responsible for the resonances in the internal lines. Eq. (17) becomes then

$$
\begin{aligned}
& \lambda=\left(\frac{4 \pi \hbar \mathrm{a}_{\text {nucl }}}{\mathrm{m}}\right)^{2}\left|\psi_{\text {rel }}(0)\right|^{2}\left(\frac{4 \pi \hbar \mathrm{a}_{\mathrm{el}}}{\mathrm{m} \sqrt{\mathrm{V}_{0}}}\right)^{2(\mathrm{~N}-1)} \\
& \quad \times \int \frac{\mathrm{d}^{2 \mathrm{~N}} \mathrm{p}}{(2 \pi)^{2 \mathrm{~N}}} \frac{2 \pi \hbar}{\mathrm{V}_{0}} \delta\left(\sum_{\mathrm{i}=1}^{2 \mathrm{~N}} \frac{\hbar^{2} \mathrm{p}_{\mathrm{i}}^{2}}{2 \mathrm{~m}_{\mathrm{i}}}-\mathrm{Q}\right) \frac{\hbar^{4}}{\mathrm{Q}^{2} \Gamma^{2}} \prod_{\mathrm{j}=1}^{\mathrm{N}-3} \frac{\mathrm{m}}{\mathrm{q}_{\mathrm{j}} \Gamma}
\end{aligned}
$$

where $\mathrm{q}_{\mathrm{j}}$ are some combinations of the remaining $2 \mathrm{~N}$ components of the final state wave vectors. Note that we have neglected the small components of the final state wave vectors in the energy delta function before the integration.

The reason, why the diagrams of the type shown in Fig 2. have the largest contribution to the transition rate, is that the wave vector combinations $\mathrm{q}_{\mathrm{j}}$ in Eq. (20) have the smallest numerical values, if the energy released in the fusion process is distributed most effectively as described above. 
Noting that the final state wave vectors are of the order $\mathrm{p}_{\mathrm{i}} \approx \sqrt{2 \mathrm{mQ} / \mathrm{N}} / \hbar$, the wave vectors $\mathrm{q}_{\mathrm{j}}$ in Eq. (20) range from $\sqrt{2 \mathrm{mQ} / \mathrm{N}} / \hbar$ to $\sqrt{\mathrm{mQ}} / \hbar$. The product in Eq. (20) can then be approximated (for the type of diagrams shown in Fig. 2):

$$
\prod_{j=1}^{\mathrm{N}-3} \frac{1}{\mathrm{q}_{\mathrm{j}}} \approx \mathrm{A}\left(\frac{\hbar^{2} \mathrm{~N}}{4 \mathrm{mQ}}\right)^{(\mathrm{N}-3) / 2}
$$

where A is some numerical constant of order unity.

In the delta function of Eq. (20) the mass corresponding to the fusion product(s) is not the deuteron mass $\mathrm{m}$. However, at the large $\mathrm{N}$ limit we can replace it also with the deuteron mass without making any essential difference. The delta function in Eq. (20) can then be written as

$$
\delta\left(\sum_{\mathrm{i}=1}^{2 \mathrm{~N}} \frac{\hbar^{2} \mathrm{p}_{\mathrm{i}}^{2}}{2 \mathrm{~m}_{\mathrm{i}}}-\mathrm{Q}\right)=\delta\left(\frac{\hbar^{2} \mathrm{p}^{2}}{2 \mathrm{~m}}-\mathrm{Q}\right)=\frac{\mathrm{m}}{\hbar^{2} \mathrm{p}} \delta\left(\mathrm{p}-\frac{\sqrt{2 \mathrm{mQ}}}{\hbar}\right) .
$$

where $\mathrm{p}$ is the length of the $2 \mathrm{~N}$ dimensional wave number vector in Eq. (20). Moreover, the $2 \mathrm{~N}$ dimensional integration volume element can be written as

$$
\begin{aligned}
\prod_{j=1}^{2 \mathrm{~N}} \mathrm{dp}_{\mathrm{j}} & =\mathrm{d}^{2 \mathrm{~N}} \mathrm{p}=\frac{2 \pi^{(2 \mathrm{~N}-1) / 2}}{\Gamma\left(\frac{2 \mathrm{~N}-1}{2}\right)} \mathrm{p}^{2 \mathrm{~N}-1} \mathrm{dp} \\
& \approx \sqrt{\frac{2 \mathrm{~N}-1}{\pi}}\left(\frac{2 \pi}{2 \mathrm{~N}-1}\right)^{(2 \mathrm{~N}-1) / 2} \mathrm{e}^{(2 \mathrm{~N}-1) / 2} \mathrm{p}^{2 \mathrm{~N}-1} \mathrm{dp}
\end{aligned}
$$

where the Stirling's approximation

$$
\Gamma(\mathrm{x}) \approx \mathrm{x}^{\mathrm{x}-1 / 2} \mathrm{e}^{-\mathrm{x}} \sqrt{2 \pi}
$$

has been used. The transition rate given by Eq. (20) becomes now

$$
\lambda \approx \mathrm{A}^{\prime}\left(\frac{4 \pi \hbar \mathrm{a}_{\text {nucl }}}{\mathrm{m}}\right)^{2}\left|\psi_{\text {rel }}(0)\right|^{2} \frac{\mathrm{e}^{\mathrm{N}}}{(4 \pi)^{\mathrm{N}}} \sqrt{\frac{\mathrm{N}^{\mathrm{N}-3}}{(2 \mathrm{~N}-1)^{2 \mathrm{~N}-2}}} \sqrt{\frac{\mathrm{Q}^{\mathrm{N}-3}\left(4 \pi \mathrm{a}_{\mathrm{el}}\right)^{4 \mathrm{~N}-4} \hbar^{2 \mathrm{~N}}}{\Gamma^{2 \mathrm{~N}-2} \mathrm{~V}_{0}^{2 \mathrm{~N}} \mathrm{~m}^{\mathrm{N}-1}}},
$$

where all the numerical factors (independent of $\mathrm{N}$ ) have been embedded in the factor $\mathrm{A}^{\prime}$. We define here a constant $\mathrm{E}^{*}$, which has the dimension of energy 


$$
\mathrm{E}^{*}=\frac{\Gamma^{2} \mathrm{~m} \mathrm{~V}_{0}^{2}}{4 \pi^{2} \mathrm{e}^{2} \mathrm{a}_{\mathrm{el}}^{4} \hbar^{2}}
$$

(Note that e in eqns. (25) and (26) is the Neper's number and not the electric charge.) By the energy constant of Eq. (26) the transition rate can be written as

$$
\lambda=\mathrm{C}\left(\frac{\hbar \mathrm{a}_{\text {nucl }}}{\mathrm{m}}\right)^{2}\left|\psi_{\text {rel }}(0)\right|^{2} \frac{\mathrm{a}_{\mathrm{el}}^{4} \hbar^{3}}{\mathrm{~N}^{2} \Gamma^{2} \mathrm{~V}_{0}^{3} \mathrm{~m}}\left(\frac{\mathrm{Q}}{\mathrm{NE}^{*}}\right)^{(\mathrm{N}-3) / 2}
$$

where all the numerical factors are now in the constant C. Moreover, $2 \mathrm{~N}-1$ in Eq. (25) have been replaced by $2 \mathrm{~N}$. This transition rate, Eq. (27), is extremely sensitive function of the trap occupation $\mathrm{N}$ and the energy released $\mathrm{Q}$. At fixed $\mathrm{N}$ the rate is a monotonic and rapidly increasing function of the released energy $\mathrm{Q}$. This shows that practically only the process having the largest possible releasing energy could take place. In the case of a fusion of two deuterons considered here, this is the ${ }^{4} \mathrm{He}$-channel, directly to the ground state. (For processes involving only two deuterons the tritium and ${ }^{3} \mathrm{He}$-channels are dominant in the usual way. So the inclusion of the trapped deuterons, besides the deuteron pairs discussed in Section 2, increase somewhat the neutron emission rate. However, for larger values of the trap occupation $\mathrm{N}$, the rates of these two-deuteron processes are vanishing small compared to the collective N-deuteron MRS-rate.)

Note that only few per cent change in the released energy (corresponding to the reaction to the first excited state) leads to the transition rate that is several orders of magnitude smaller than the transition rate to the grounds state even for moderate values of the trap occupation $\mathrm{N}$.

As the dominant fusion process will be $\mathrm{d}+\mathrm{d} \rightarrow{ }^{4} \mathrm{He}$ (to the ground state and without any final state photon) there will be no radiation (except possible soft secondary radiation caused by the free deuterons in the final state having kinetic energies typically in $\mathrm{keV}$ range).

When the trap occupation $\mathrm{N}$ increases, the rate given by Eq. (27) increases by two ways. First, there is the rapid change arising from the factor

$$
\mathrm{f}(\mathrm{N})=\left(\frac{\mathrm{Q}}{\mathrm{NE}^{*}}\right)^{(\mathrm{N}-3) / 2}
$$

Second, the overlap of the deuteron wave functions $\left|\psi_{\text {rel }}(0)\right|^{2}$ increases, when the density of the deuteron gas in the trap increases. If we consider the fusion rate as a function of the trap volume $\mathrm{V}_{0}$, we note that there will be a "window", where the fusion can take place. If the trap volume is too small (the trap consisting only one or very few surface atoms), the trap cannot maintain such a high deuteron occupation that the factor $f(N)$ could be large enough to produce detectable transition rates in Eq. (27). On the other hand, for 
very large trap volumes the deuteron gas in the trap remains dilute (so that the nuclear overlapping factor $\left|\psi_{\text {rel }}(0)\right|^{2}$ remains small) until the factor $\mathrm{f}(\mathrm{N})$ has passed already its maximum value and is decreasing preventing the fusion to occur.

However, if the trap volume is in the fusion "window", the ${ }^{4} \mathrm{He}$-fusion occurs inevitably, when $\mathrm{N}$ become large enough. As a matter of fact, the fusion rate therefore depends ultimately on the rate by which the occupation increases in these traps.

It should be noted that larger released energies are obtained in multi deuteron processes (with two or more deuterons reacting with a heavier nucleus). The expression for the rate in these transmutation processes, corresponding to Eq. (27), contains more than one nuclear overlapping factor $\left|\psi_{\text {rel }}(0)\right|^{2}$. The overlapping of a deuteron and a heavier nucleus depends similarly on the deuteron density in the trap, as the overlapping of two deuterons, although less sensitively. Similarly, as for the fusion reaction, there are "windows" in the trap volume for the different transmutation processes. At larger volumes the transmutation processes are more probable than the fusion process due to the larger releasing energy and the smaller value of the deuteron overlapping.

What transmutation reactions can really occur, depends then on the released energy Q, the nuclear charge number $\mathrm{Z}$, the trap volume $\mathrm{V}_{0}$ and the scattering lengths. There are large uncertainties in the numerical values corresponding to the trap volume and to the elastic scattering length and also to the line width. These uncertainties prevent us from predicting the relative transition rate of the transmutation processes to that of the ${ }^{4} \mathrm{He}$ fusion process. Anyway, we can understand by this model that the ratio of the excess heat to the excess ${ }^{4} \mathrm{He}$ varies depending on the volumes of the traps.

If light water is used instead of the heavy water, the surface atoms can similarly trap the protons. In the case of light water, however, there will be no proton-proton fusion, because the energy released is too low. Anyway, transmutation processes with large released energy can take place.

We can also expect to obtain fusion reactions and/or transmutation processes in other type of experiments (for example, gas permeation experiments etc.) provided that deuteron traps, similar as here, emerge. 


\section{Conclusions and Outlook}

We assumed that in the electrolysis of heavy water the deuterium ions reaching the cathode form (together with the electrons around) a deuterium plasma in the surface region of the host metal. We calculated the effective potential at one loop level with energy spectrum of free particles. With these inputs we found that the deuterons experience an attraction potential, which sustain bound states for deuteron pairs. The equilibrium separation of the deuterons in this effective potential is of the order that allow the fusion processes to occur with low but detectable rate for the neutron emission.

Furthermore, we assumed that the surface atoms trap deuterons by this effective potential. The fusion processes in these traps were considered assuming that the trapped deuterons collectively share the released energy becoming free particles. A simplified model with general argument was used to extract the main physical consequences of the processes. It was shown that the rate of the nuclear process increases very rapidly with the releasing energy showing that the ${ }^{4} \mathrm{He}$-channel is in practice the only emerging fusion channel. Moreover, we argued that transmutation processes having similarly large releasing energies occur.

For further research a more refined model for the deuteron trap is required and the ground state in this trap has to be solved. Moreover, the numerical uncertainties corresponding to the N-particle scattering matrix element has to be reduced in order to obtain accurate predictions of the relative rates for the fusion and transmutation processes. In order to get an estimate for the absolute reaction rates, better knowledge is required on the details of the flow dynamics and the surface structure and their influence to the trap formation and to the trap occupation rate.

Acknowledgements

I would like to thank Carl-Gustav Källman for valuable discussions and comments.

\section{References:}

[1] M. Fleischmann, S. Pons and M. Hawkins, J. Electroanal. Chem. 261 (1989) 301

[2] T. Toimela, to appear

[3] A. L. Fetter and J. D. Walecka, Quantum Theory of Many-Particle Systems, (McGraw-Hill, 1971)

[4] S. Feng, Solid State Commun. 72 (1989) 205

[5] E. K. Storms, Infinite Energy 1 (1996) 7

[6] K. Czerski, A. Huke, P. Heide and G. Ruprecht, Europhys. Lett. 68 (2004) 363

[7] William A. Fowler, Georgeanne R. Caughlan and Barbara A. Zimmerman, Annu. Rev. Astron. Astrophys. 5 (1967) 525

[8] S. E. Jones, E. P. Palmer, J. B. Czirr, D. L. Decker, G. L. Jensen, J. M. Thorne, S. F. Taylor and J. Rafelski, Nature 388 (1989) 73 